Meta

Journal des traducteurs

Translators' Journal

\title{
Pour ne pas filer un mauvais coton...
}

\section{Marthe Faribault}

Volume 19, numéro 2, juin 1974

URI : https://id.erudit.org/iderudit/002726ar

DOI : https://doi.org/10.7202/002726ar

Aller au sommaire du numéro

Éditeur(s)

Les Presses de l'Université de Montréal

ISSN

0026-0452 (imprimé)

1492-1421 (numérique)

Découvrir la revue

Citer cet article

Faribault, M. (1974). Pour ne pas filer un mauvais coton... Meta, 19(2), 90-97.

https://doi.org/10.7202/002726ar d'utilisation que vous pouvez consulter en ligne.

https://apropos.erudit.org/fr/usagers/politique-dutilisation/ 


\section{POUR NE PAS FILER UN MAUVAIS COTON...}

Le vocabulaire des textiles, très riche en raison de l'abondance des matériaux, des techniques et des produits, présente, comme bien des corps de vocabulaire technique, une pléthore de synonymes ainsi que de nombreux cas de polysémie, ce qui en complique singulièrement l'étude. Ainsi, pour le vocabulaire de la préparation du coton en filature ${ }^{1}$, que nous allons aborder, la machine qui a pour nom anglais vertical opener s'appelle indifféremment en français ouvreuse à axe vertical, à arbre vertical ; ouvreuse Craighton, ou Crighton ou de Crighton; ouvreuse Crighton à arbre vertical et enfin ouvreuse verticale. Même si toutes ces expressions sont apparentées, on se demande comment le profane pourra s'y retrouver.

L'ouvreuse fait partie d'un ensemble de machines appelé ligne d'ouverture ou Train d'ouvraison (opening line), dont le rôle est de démêler, mélanger, nettoyer le coton et le mettre sous la forme d'une nappe. Cette étape, suivie du cardage dont nous ne parlerons pas dans le présent article, est indispensable pour l'obtention d'un fil régulier et propre sur le métier à filer. On peut également préparer le coton dans une machine unique, qui conjugue les différentes machines de la ligne d'ouverture et qui porte le nom de one-process en français (single process picker assembly). Il n'existe malheureusement pas à l'heure actuelle d'équivalent français

1. Ce corps de vocabulaire a été étudié dans le cadre d'un stage à la Banque de terminologie de l'Université de Montréal, dirigé par M. Robert Dubuc, au cours de l'été 1973. 
pour cette machine. Les agencements possibles de machines dans la ligne d'ouverture sont extrêmement nombreux. De plus, selon la propreté et la finesse du coton, il peut s'avérer avantageux d'éviter certaines machines; on équipe donc souvent les lignes d'ouverture de by-pass, ou mieux dit, de dérivations (by-passes), comme le propose le Comité d'étude des termes techniques français. Nous allons maintenant étudier en détail les différentes machines d'une ligne d'ouverture type et voir les problèmes terminologiques qui peuvent se poser.

Il s'agit d'abord de défaire les balles de coton en gros flocons (fig. 1). On se sert à cette fin de tables d'aiguilles, ou tablettes à aiguilles (pluckers), qui arrachent le coton par touffes sous les balles. L'ensemble de ces tables s'appelle le raboteur de balles, qu'on rendra en anglais par le pluriel pluckers. Mais cette opération est très souvent pratiquée à la main. L'ouvrier prélève sur plusieurs balles des touffes de coton et les dépose, comme les tablettes à aiguilles le font, sur un tablier alimentaire à lattes (lattice conveyer apron) qui est le premier élément d'une machine appelée chargeuse ou chargeuse-mélangeuse (blending feeder; cotton feeder; feeder). Ce tablier amène les touffes au pied d'un tablier élévateur, ou tablier à pointes (lifting apron; lift apron; spiked apron; spike apron; pin apron) qui défait les touffes en flocons plus petits. À l'extrémité supérieure du tablier élévateur se trouve une ouvreuse de fibres (en anglais, comb, s'il s'agit d'une petite table d'aiguilles, et combing roll, s'il s'agit d'un cylindre à pointes), qui détache les flocons trop gros. Les autres flocons, pris entre les pointes du tablier élévateur, continuent leur chemin et sont arrachés par un détacheur (stripping roll; doffer; stripper roll) qui les projette dans une trémie (hopper). Cette dernière décharge la matière sur une bande transporteuse, ou ruban transpor-

\section{FIGURE 1}

Ouverture des balles

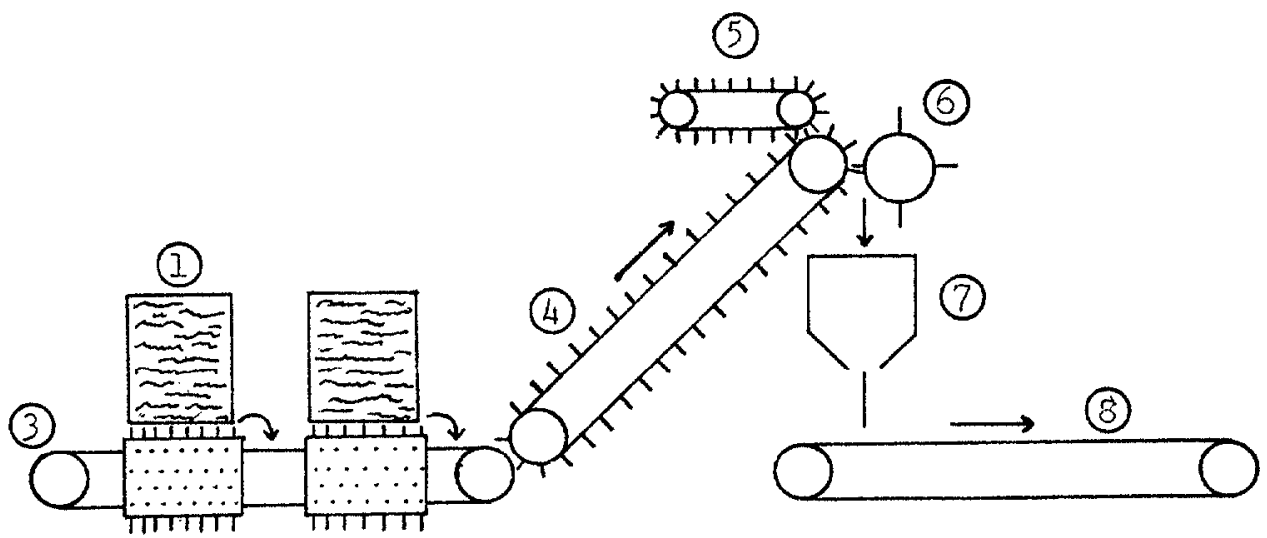

(2)

1. balle de coton

5. ouvreuse de fibres

2. tablette d'aiguilles

6. détacheur

3. tablier alimentaire à lattes

7. trémie

4. tablier à pointes

8. table de transport 
teur, bande de transport, table de transport (conveyer belt; conveying apron system ; conveyer apron). Si cette bande transporteuse recueille la matière provenant de plusieurs chargeuses, elle s'appellera tablier collecteur. Outre l'ouverture des balles, cet ensemble de machines effectue un mélange (en anglais blending et plus rarement mixing) des flocons provenant des différentes balles, ce qui assure une certaine homogénéité de la matière et, par conséquent, favorise l'obtention de fils réguliers au métier à filer.

FTGURE 2a Ouvreuse Crighton

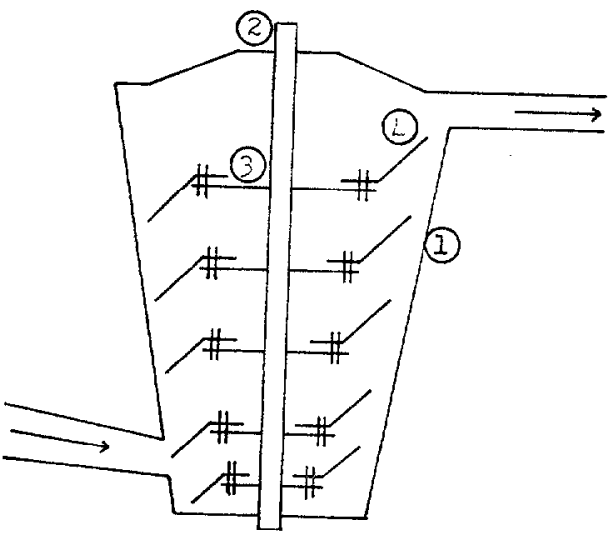

1. cage conique

2. arbre

3. plateau

4. couteau
FIGURE 2b Ouvreuse Buckley

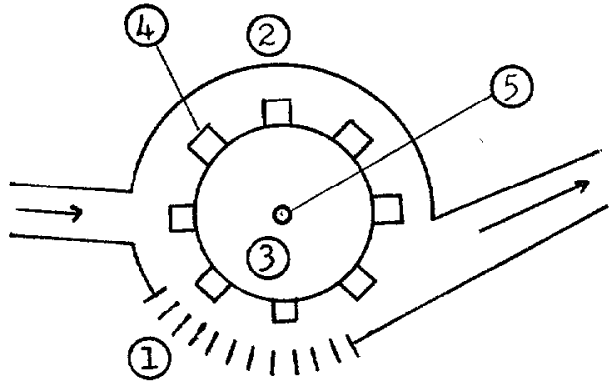

1. grilles périphériques

2. cage périphérique

3. disque

4. couteau

5. arbre ensemble tournan

FIGURE $2 c$

Ouvreuse en cascades

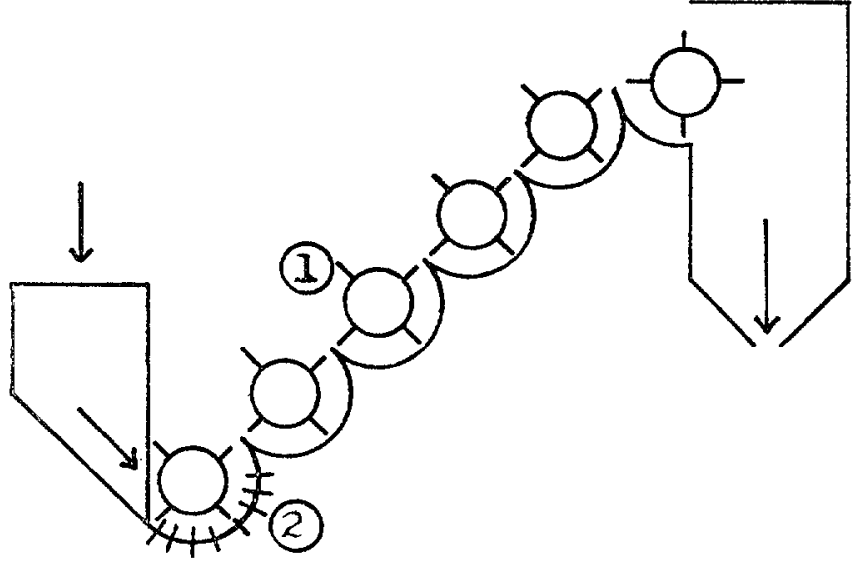

1. tambour à broches 
L'usage des termes relatifs à ces premières machines est mouvant. En effet, si le coton est prélevé à la main sur les balles et qu'il est déposé, toujours à la main, sur le tablier alimentaire à lattes de la chargeuse, cette dernière est considérée comme brise balles, ou brise-balle, floconneuse, ouvreuse de balles, ouvreuse (bale breaker). Par contre, si le coton est prélevé à la machine au moyen de tables d'aiguilles, ou raboteur de balles, ce dernier sera considéré comme le brise-balle, mais on ne saurait dans ce cas utiliser les synonymes floconneuse, ouvreuse de balles et ouvreuse. D'autre part, l'ensemble tablier alimentaire, tablier élévateur, ouvreuse de fibres, détacheur et trémie est désigné dans ce cas sous le nom de chargeuse, ou chargense-mélangeuse (blending feeder; feeder; cotton feeder). Il existe également sur le marché une machine unique qui réunit le raboteur de balles et la chargeuse ; elle se vend sous le nom de multi-floconneuse (Multi-tuft blender).

Le rôle de ces premières machines est donc d'ouvrir la balle : c'est l'étape dite du mélange-onverture, ou floconnage, mélange-effillochage (bale opening ; opening). Les termes floconnage et mélange-effilochage ne s'appliquent cependant que dans le cas où les balles sont ouvertes par le raboteur de balles, tandis que mélangeouverture s'applique indifféremment dans les deux cas.

Les flocons provenant des balles ouvertes dans l'opération précédente sont ensuite acheminés vers une machine appelée ouvreuse (opener; opening machine), qui ouvre davantage le coton tout en le nettoyant. On parle souvent de ces machines comme de machines de nettoyage (cleaning machines; cleaners), car le nettoyage est l'un de leurs rôles. Les ouvreuses les plus couramment utilisées sont l'ouvreuse Crighton (fig. 2a), qui s'appelle en anglais vertical opener et dont nous avons déjà parlé, et l'ouvreuse Buckley (fig. 2b) ou ouvreuse à grand tambour horizontal (Buckley opener). De conception plus récente, l'ouvreusenettoyeuse à étages ou ouvreuse inclinée, épurateur à échelons, nettoyeuse en cascades (fig. 2c), mieux connue sous le nom anglais de Superior Cleaner, qui est une marque de commerce, est composée principalement d'une ligne de cylindres à broches inclinées. L'Axiflo, ou Axi-Flo opener cleaner en anglais (encore une marque de commerce), est une ouvreuse à tambour horizontal, mais de conception plus moderne que l'ouvreuse Buckley. L'élément principal de ces machines est appelé beater en anglais, terme qui se rend en français par les expressions ensemble tournant, s'il s'agit des ouvreuses Crighton et Buckley, et par tambour à broches, s'il s'agit de l'ouvreuse en cascades.

Le coton, après avoir passé ce deuxième stade de la préparation en filature, c'est-à-dire l'ouverture proprement dite de la matière (opening), s'achemine vers la dernière machine de la ligne d'ouverture : le batteur nappeur (fig. 3), ou batteur (picker ou, plus rarement, scutcher). Cette machine procède à une ouverture plus complète de la matière et débite une nappe de fibres, appelée nappe de batteur (picker lap) qui est enroulée sur une tringle pour former le rouleau de batteur (lap). C'est le stade dit du battage nappage, ou battage (picking). Le batteur nappeur se compose, pour l'ouverture et le nettoyage du coton, d'un volant batteur (bar beater; beater) composé d'un axe supportant des bras radiaux 
FIGURE 3

Batteur nappeur

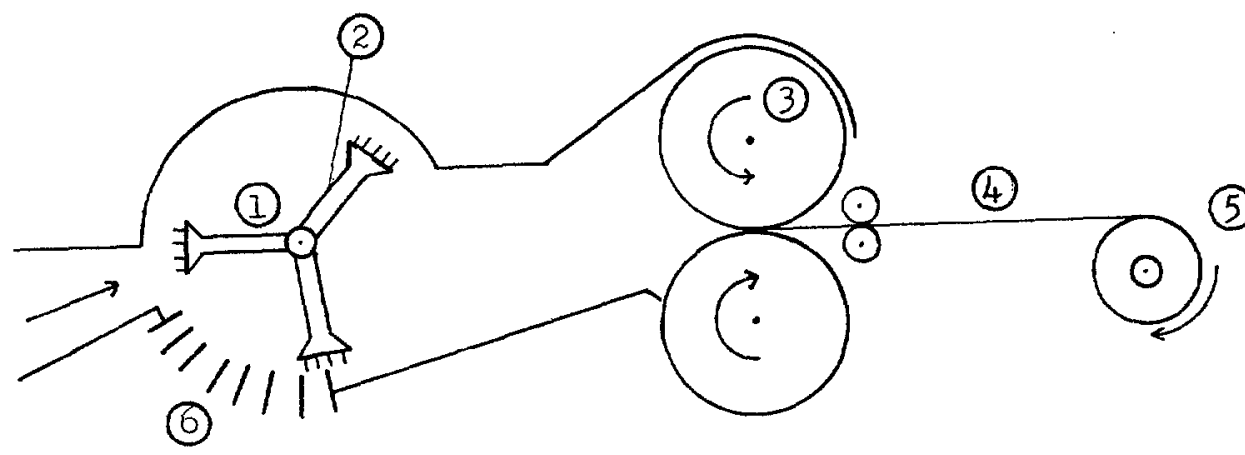

1. volant Kirschner
2. bras
3. tambour perforé

4. nappe de batteur

5. rouleau de batteur

6. grille périphérique

sur lesquels sont fixées deux ou trois règles ou battes (lag) ou d'un volant Kirschner ou volant cardeur (Kirschner beater; carding beater), qui est identique au volant batteur, sauf pour les règles qui sont remplacées par des pointes. Les fibres sont ensuite plaquées par un courant d'air sur des tambours perforés (screen sections; screens) et se forment ainsi en nappe. Le coton est alors prêt pour le cardage.

Jusqu'ici l'exposé rend compte des termes employés par plusieurs auteurs. Avant de conclure, il serait intéressant de s'arrêter pour étudier la terminologie très particulière d'un auteur ${ }^{2}$ consulté pour cet article. Il s'agit de la description d'une ligne d'ouverture : «Opérations préparatoires» (à la filature du coton) «Ouvrage-battage. Le but est de desserrer et de nettoyer le coton. Ce dernier passe dans une suite de $«$ batteurs $»$ :

- le «batteur-ouvreur» ou «brise-balles » disloque la masse compacte ;

- le « batteur-éplucheur» la divise encore et la débarrasse des débris ;

- le «batteur-étaleur» achève la division et transforme le coton, devenu vaporeux, en une nappe légère, propre, mais à fibres toujours enchevêtrées. 》 Vient ensuite le cardage.

Cette solution, de prime abord, peut sembler heureuse. En effet, elle regroupe toutes les machines de la ligne d'ouverture sous un générique, soit le mot « batteur », qu'elle fait suivre d'un spécifique qui particularise chacune des machines. Cependant, cette démarche appelle les restrictions suivantes : d'abord, nous avons affaire à des mots composés; or il est très courant dans ce cas de ne retenir qu'un élément du mot. C'était d'ailleurs le cas pour le batteur nappeur de notre exposé,

2. $\mathbf{M}^{\mathrm{me}} \mathrm{M}$. Le Fustec, Technologie des matières et industries textiles, Paris, Eyrolles, 1970, p. 178. 
appelé couramment batteur. Nous nous retrouverions alors devant trois machines totalement différentes, qui porteraient toutes trois 1'appellation de «batteur ». De plus, si le terme «batteur» s'applique bien dans le cas du «batteur-éplucheur» (l'ouvreuse de notre exposé) et du «batteur-étaleur» (le batteur nappeur de notre exposé), car il y a effectivement battage du coton dans ces machines, il n'a cependant aucun rapport avec l'action que subit le coton dans le « batteurouvreur» (le brise-balles de notre exposé) ; en effet, dans cette machine il y a arrachage du coton par les tables d'aiguilles et malaxage de ces fibres dans la chargeuse, mais jamais de battage à proprement parler. D'autre part, les spécifiques employés par cet auteur sont d'une propriété douteuse, en particulier les expressions «batteur-ouvreur» et «batteur-éplucheur». En effet, d'après ce que nous avons déjà vu de ces machines, le terme «éplucheur » s'appliquerait davantage à la réalité du brise-balles ; d'autre part le terme "ouvreur» serait acceptable pour les deux machines, mais conviendrait davantage à l'ouvreuse, puisque l'ouverture est la fonction spécifique de cette machine. Quant au terme «étaleur », il est très correct; ce serait un synonyme plus abstrait de «nappeur ». Enfin, l'expression " ouvrage-battage » nous semble à proscrire, à cause du manque absolu de convenance du mot ouvrage à l'action désignée.

Cette critique peut sembler quelque peu sévère, mais il faut tenir compte qu'il s'agit d'une terminologie particulière à un auteur, qui présente certes des avantages mais qui introduit beaucoup d'inexactitudes, en plus de venir en concurrence avec un usage relativement bien établi.

Pour en revenir à notre description de la ligne d'ouverture, qui rend compte de l'usage courant, nous voyons, outre les problèmes terminologiques déjà discutés en cours d'exposé, que le terme "beater» est polysémique. Dans le cas des batteurs nappeurs, beater a un sens générique, qu'on traduira par volant, ou par volant batteur et volant Kirschner, ou volant cardeur, si l'on veut spécifier.

Enfin les termes relatifs aux fonctions de la ligne d'ouverture présentent un problème. Le terme ouverture (opening), tant en anglais qu'en français, s'applique à l'ouverture des balles, en corrélation avec les spécifiques français floconnage, mélange-effilochage et mélange-ouverture, et bale opening en anglais. Il s'applique plus spécifiquement au rôle joué par l'ouvreuse, avec pour synonyme ouvraison, qu'il nous semble préférable d'éviter à cause de son homonyme courant dérivé du verbe ouvrer. Il désione encore le rôle rempli par le volant du batteur nappeur, qu'on appelle plus souvent battage. Et enfin, il s'applique au rôle général de la ligne d'ouverture, en corrélation avec le spécifique composé ouvraisonbattage, ou mieux, ouverture-battage, qu'on rendrait en anglais par l'expression opening and picking.

Marthe Faribault

\section{BIBLIOGRAPHIE}

Brossard, I., les Fils et les tissus, $2^{\mathbf{e}}$ éd., Paris, Dunod, 1971, 93 p.

Brossard, I., Technologie des textiles, Paris, Dunod, 1970, 296 p. 
Colin, F., Principes généraux de la filature, Paris, Librairie polytechnique Béranger, 1964, $148 \mathrm{p}$.

De Calan, Pierre, le Coton et l'industrie cotonnière, $2^{\mathrm{e}}$ éd. revue et mise à jour, Paris, P.U.F., "Que sais-je ? ", n 90, 1966, 126 p.

Hamby, D. S., The American Cotton Handbook, $3^{\mathrm{e}}$ éd., New York, Interscience Publishers, 1965 , vol. 1 .

Le Fustec, Mme M., Technologie des matières et industries textiles, $5^{\mathrm{e}}$ éd., Paris, Eyrolles, $1970,191 \mathrm{p}$.

L'Industrie textile, revue mensuelle, Paris, septembre 1971 et janvier 1972.

Linton, G.E., The Modern Textile Dictionary, New York, Meredith Publishing Co., 1963, $1077 \mathrm{p}$.

Marchal, Lucien, l'Or blanc. La prodigieuse aventure du coton, Bruxelles, Marabout, « Marabout Université $30 », 1963,284 \mathrm{p}$.

Stout, Evelyn F., Introductory to textiles, 3e éd., Holt Rinehart and Winston, 1966, 455 p.

Thiébaut, Raymond, la Filature, 2e éd., Paris, P.U.F., "Que sais-je ? », nº 537, 1964, 126 p.

Axi-Flo opener cleaner

bale breaker

bale opening; opening

bar beater; beater

beater

blending; mixing

blending feeder; cotton feeder; feeder

Buckley opener

by-pass

cleaning machine; cleaner

comb

combing roll

conical casing; beater chamber

conveyer belt; conveying apron system; conveyer apron

cylindrical chamber

doffer; stripping roll; stripper roll

finger

\section{Axiflo}

brise balles; brise-balle

floconneuse

ouvreuse de balles; ouvreuse

floconnage

mélange-ouverture mélange-effilochage

volant batteur; volant

ensemble tournant tambour à broches

mélange

chargeuse-mélangeuse; chargeuse

ouvreuse Buckley; ouvreuse à grand tambour horizontal; ouvreuse à axe horizontal

dérivation; bi-pass

machine de nettoyage

ouvreuse de fibres

ouvreuse de fibres

cage conique; grilles périphériques

bande transporteuse; ruban transporteur bande de transport; table de transport; tablier collecteur

cage périphérique

détacheur

conteau 


grid bars; grids
grid section
hopper
Kirschner beater; carding
beater
lag
lap
lattice conveyer apron
lifting apron; lift apron
Multi-tuft blender
opener; opening machine
opening
opening and picking
opening line
picker; scutcher
picker lap
picking
plate
plucker
pluckers
screen section; screen
single-process picker
assembly
spiked apron; spike apron;

grille périphérique (ouvreuse Crighton)

grille

trémie

volant Kirschner; volant cardeur

règle; batte

rouleau de batteur

tablier alimentaire à lattes

tablier élévateur

Multi-floconneuse

ouvreuse

ouverture; ouvraison

ouvraison-battage; ouverture-battage

ligne d'ouverture; train d'ouvraison

batteur nappeur; batteur

nappe de batteur

battage nappage; battage

plateau; disque circulaire; disque

table d'aiguilles; tablette à aiguilles

raboteur de balles

tambour perforé

One-Process

One-Process

tablier à pointes

épurateur à échelons; nettoyeuse en cascades; ouvreuse inclinée; ouvreuse-nettoyeuse à étages

ouvreuse à axe vertical; ouvreuse Crighton; ouvreuse Crighton à arbre vertical; ouvreuse Cright; ouvreuse verticale; ouvreuse de Crighton; ouvreuse à arbre vertical 